\title{
Application of Optimal Homotopy Asymptotic Method to Some Well-Known Linear and Nonlinear Two-Point Boundary Value Problems
}

\author{
Muhammad Asim Khan $\mathbb{D}^{1},{ }^{1}$ Shafiq Ullah, ${ }^{2}$ and Norhashidah Hj. Mohd Ali ${ }^{1}$ \\ ${ }^{1}$ School of Mathematical Sciences, Universiti Sains Malaysia, 11800 Penang, Malaysia \\ ${ }^{2}$ Department of Mathematics, University of Peshawar, Pakistan \\ Correspondence should be addressed to Muhammad Asim Khan; asim.afg@gmail.com
}

Received 20 May 2018; Accepted 21 October 2018; Published 3 December 2018

Guest Editor: Dongfang Li

Copyright (C) 2018 Muhammad Asim Khan et al. This is an open access article distributed under the Creative Commons Attribution License, which permits unrestricted use, distribution, and reproduction in any medium, provided the original work is properly cited.

The objective of this paper is to obtain an approximate solution for some well-known linear and nonlinear two-point boundary value problems. For this purpose, a semianalytical method known as optimal homotopy asymptotic method (OHAM) is used. Moreover, optimal homotopy asymptotic method does not involve any discretization, linearization, or small perturbations and that is why it reduces the computations a lot. OHAM results show the effectiveness and reliability of OHAM for application to two-point boundary value problems. The obtained results are compared to the exact solutions and homotopy perturbation method (HPM).

\section{Introduction}

Two-point boundary value problems (TPBVP) have many applications in the field of science and engineering [1,2]. These problems arise in many physical situations like modeling of chemical reactions, heat transfer, viscous fluids, diffusions, deflection of beams, the solution of optimal control problems, etc. Due to the wide applications and importance of boundary value problems (BVP) in science and engineering we need solutions to these problems.

There are many techniques available for the solution ofof BVP like Adomian Decomposition Method (ADM) [37], Extended Adomian Decomposition Method (EADM) [8], Differential Transformation Method (DTM) [9], Variational Iteration Method (VIM) [10], Perturbation methods(PMs) $[1,11-13]$, and so on. Perturbation methods are easy to solve but they require small parameters which are sometimes not an easy task. Recently V. Marinca et al. presented optimal homotopy asymptotic method (OHAM) [14] for the solution of BVP, which did not require small parameters. The method can also be applied to solve the stationary solution of some partial differential equations, e.g., gKdv equation, nonlinear parabolic problems, and so on [15-20]. In OHAM, the concept of homotopy is used together with the perturbation techniques. Here, OHAM is applied to TPBVP to check the applicability of OHAM for TPBVP.

\section{Basics of OHAM}

Let us take the BVP whose general form is the following:

$$
\begin{gathered}
\mathscr{L}(w(\xi))+\mathbb{N}(w(\xi))+F(\xi)=0, \\
B\left(w, \frac{d w}{d \xi}\right)
\end{gathered}
$$

where $\mathscr{L}$ is a linear operator, $\xi$ is independent variable, $\mathbb{N}$ is the nonlinear operator, $F(\xi)$ is a known function, and $B$ is a boundary operator.

Homotopy on OHAM can be constructed as

$$
\begin{aligned}
& (1-\mathrm{p})(\mathscr{L}(\varphi(\xi, \mathrm{p})+F(\xi)) \\
& \quad=H(\mathrm{p})(\mathscr{L}(\varphi(\xi, \mathrm{p})+F(\xi)+\mathbb{N}(\varphi(\xi, \mathrm{p}))), \\
& B\left(\varphi(\xi, \mathrm{p}), \frac{\partial \varphi(\xi, \mathrm{p})}{\partial \xi}\right)
\end{aligned}
$$


where $\mathrm{p} \in[0,1]$ is an embedding parameter, $\varphi(\xi, \mathrm{p})$ is an unknown function, $H(\mathrm{p})$ is a nonzero auxiliary function for $\mathrm{p} \neq 0$, and $H(\mathrm{p})$ is of the form

$$
H(\mathrm{p})=\mathrm{p} \mathbb{C}_{1}+\mathrm{p}^{2} \mathbb{C}_{2}+\mathrm{p}^{3} \mathbb{C}_{3}+\cdots
$$

Clearly when $\mathrm{p}=0$ then $H(0)=0$. And obviously, when $\mathrm{p}=0$ then $\varphi(\xi, 0)=w_{0}(\xi)$. When $\mathrm{p}=1$ then $\varphi(\xi, 1)=w(\xi)$. So as p increases from 0 to 1 , the solution $\varphi(\xi, \mathrm{p})$ varies from $w_{0}(\xi)$ to the exact solution $w(\xi)$, where $w_{0}(\xi)$ is obtained from (2) for $\mathrm{p}=0$

$$
\mathscr{L}\left(w_{0}(\xi)+\digamma(\xi)=0\right.
$$

The proposed solution of (1) will be of the form

$$
\varphi\left(\xi, \mathrm{p}, \mathbb{C}_{i}\right)=w_{0}(\xi)+\sum_{k \geq 1} w_{k}\left(\xi, \mathbb{C}_{i}\right) \mathrm{p}^{k}, \quad i=1,2,3, \ldots
$$

Substituting this value of $\varphi\left(\xi, \mathrm{p}, \mathbb{C}_{i}\right)$ into (1), after some calculations, we can obtain the governing equations of $w_{0}(\xi)$ by using (4) and $w_{k}(\xi)$, that is,

$$
\begin{aligned}
& \mathscr{L}\left(w_{1}(\xi)\right)=\mathbb{C}_{1} \mathbb{N}_{0}\left(w_{0}(\xi)\right), \\
& B\left(w_{1}, \frac{d w_{1}}{d \xi}\right)=0 \\
& \mathscr{L}\left(w_{k}(\xi)-w_{k-1}(\xi)\right) \\
& \quad=\mathbb{C}_{k} \mathbb{N}_{0}\left(w_{0}(\xi)\right)+\sum_{i=1}^{k-1} \mathbb{C}_{i} \mathscr{L}\left(w_{k-1}(\xi)\right. \\
& \quad+\mathbb{N}_{k-1}\left(w_{0}(\xi), w_{1}(\xi), w_{2}(\xi), \ldots, w_{k-1}(\xi)\right), \\
& B\left(w_{k}, \frac{d w_{k}}{d \xi}\right)=0,
\end{aligned}
$$

where $\mathbb{N}_{m}\left(w_{0}(\xi), w_{1}(\xi), w_{2}(\xi), \ldots, w_{m}(\xi)\right)$ is the coefficient of $\mathrm{p}^{m}$ in the series expansion of $\mathbb{N}\left(\varphi\left(\xi, \mathrm{p}, \mathbb{C}_{i}\right)\right)$ with respect to the embedding parameter $\mathrm{p}$. And

$$
\begin{array}{r}
\mathbb{N}\left(\varphi\left(\xi, \mathrm{p}, \mathbb{C}_{i}\right)\right)=\mathbb{N}_{0}\left(w_{0}(\xi)\right) \\
+\sum_{m \geq 1} \mathbb{N}_{m}\left(w_{0}, w_{1}, w_{2}, \ldots, w_{m}\right) \mathrm{p}^{m}, \\
i=1,2,3, \ldots, m
\end{array}
$$

where $\varphi\left(\xi, \mathrm{p}, \mathbb{C}_{i}\right)$ is given by (5). The convergence of series (5) depends on the convergence of the constants $\mathbb{C}_{i}^{\prime} s$, if these constants are convergent at $\mathrm{p}=1$, then the solution becomes

$$
w\left(\xi, \mathbb{C}_{i}\right)=w_{0}(\xi)+\sum_{k \geq 1}\left(w_{k}\left(\xi, \mathbb{C}_{i}\right)\right) .
$$

Generally, the mth order solution of the problem can be obtained in the form

$$
w^{(m)}\left(\xi, \mathbb{C}_{i}\right)=w_{0}(\xi)+\sum_{k=1}^{m}\left(w_{k}\left(\xi, \mathbb{C}_{i}\right)\right)
$$

Putting this solution in (1) we get the following residual:

$$
\begin{aligned}
\mathfrak{R}\left(\xi, \mathbb{C}_{i}\right)= & \mathscr{L}\left(w^{(m)}\left(\xi, \mathbb{C}_{i}\right)+F(\xi)\right) \\
& +\mathbb{N}\left(w^{(m)}\left(\xi, \mathbb{C}_{i}\right)\right), \quad i=1,2,3, \ldots, m
\end{aligned}
$$

If $\mathfrak{R}\left(\xi, \mathbb{C}_{i}\right)=0$, then the solution is going to be exact, but generally, such a situation does not arise in nonlinear problems but the functional defined below can be minimized

$$
J\left(\xi, \mathbb{C}_{i}\right)=\int_{x_{0}}^{x_{1}} \Re^{2}\left(\xi, \mathbb{C}_{i}\right) d \xi,
$$

where $x_{0}$ and $x_{1}$ are two constants depending on the given problem. The values of $\mathbb{C}_{i}^{\prime} s$ can be optimally found by the condition

$$
\frac{\partial J}{\partial \mathbb{C}_{1}}=\frac{\partial J}{\partial \mathbb{C}_{2}}=\cdots=\frac{\partial J}{\partial \mathbb{C}_{m}}=0
$$

After knowing these constants, the solution (10) is well determined.

\section{Examples}

To check the applicability of OHAM for TPBVP, in this section four examples of TPBVP are presented in which one example is linear and the remaining are nonlinear.

3.1. Example 1. Let us consider the linear problem [1] of second order

$$
\begin{aligned}
w^{\prime \prime}(\xi) & =w^{\prime}(\xi)-e^{(\xi-1)}-1, \quad 0<\xi<1, \\
w(0) & =0, \\
w(1) & =1
\end{aligned}
$$

The exact solution of problem (14) is $\xi\left(1-e^{\xi-1}\right)$. Now according to OHAM $\mathscr{L}\left(w_{0}(\xi)\right)=w^{\prime \prime}(\xi)-w^{\prime}(\xi)$, the nonlinear part $\mathbb{N}(w(\xi))=0$ and $F(\xi)=e^{(\xi-1)}+1$.

The zeroth-order problem is

$$
\begin{aligned}
w_{0}^{\prime \prime}(\xi)-w_{0}^{\prime}(\xi) & =1+e^{\xi-1}, \\
w_{0}(0) & =0, \\
w_{0}(1) & =1
\end{aligned}
$$

The solution of (15) is

$$
w_{0}(\xi)=\frac{\left(e-e^{\xi}\right) \xi}{\xi}
$$

The first-order problem is

$$
\begin{aligned}
w_{1}^{\prime \prime}(\xi)-w_{1}^{\prime}(\xi)= & -1-e^{\xi-1}-\mathbb{C}_{1}-\mathbb{C}_{1} e^{\xi-1}+w_{0}^{\prime}(\xi) \\
& +\mathbb{C}_{1} w_{0}^{\prime}(\xi)-w_{0}^{\prime \prime}(\xi) \\
& -\mathbb{C}_{1} w_{0}^{\prime \prime}(\xi), \\
w_{1}(0)= & 0 \\
w_{1}(1)= & 0 .
\end{aligned}
$$


TABLE 1: Comparison of the third-order OHAM solution with the exact solution and HPM.

\begin{tabular}{lcccc}
\hline$\xi$ & OHAM Solution $\left(w^{(2)}\right)$ & Exact & HPM [1] & $\left|w^{(2)}-E x a c t\right|$ \\
\hline 0.1 & 0.059343 & 0.059343 & 0.05934820 & $1.38778 \times 10^{-17}$ \\
\hline 0.3 & 0.151024 & 0.151024 & 0.15103441 & $2.77556 \times 10^{-17}$ \\
\hline 0.5 & 0.196735 & 0.196735 & 0.19673826 & $2.77556 \times 10^{-17}$ \\
\hline 0.7 & 0.181427 & 0.181427 & 0.18142196 & $5.55112 \times 10^{-17}$ \\
\hline 0.9 & 0.0856463 & 0.0856463 & 0.08564186 & $5.55112 \times 10^{-17}$ \\
\hline
\end{tabular}

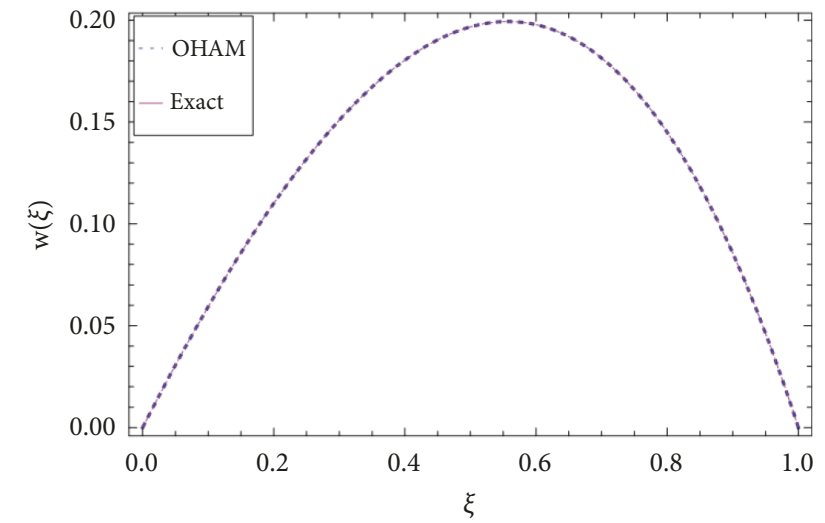

Figure 1: Comparison between exact solution (dashed line) and approximate solution (dotted line) for example 1.

The solution of (17) is

$$
w_{1}(\xi)=0
$$

The second-order problem is

$$
\begin{aligned}
w_{2}^{\prime \prime}(\xi)= & -\mathbb{C}_{2}-\exp (\xi-1) \mathbb{C}_{2}+\mathbb{C}_{2} w_{0}^{\prime}(\xi)+w_{1}^{\prime}(\xi) \\
& +\mathbb{C}_{1} w_{1}^{\prime}(\xi)-w_{2}^{\prime}(\xi)-\mathbb{C}_{2} w_{0}^{\prime \prime}(\xi)-w_{1}^{\prime \prime}(\xi) \\
& -\mathbb{C}_{1} w_{1}^{\prime \prime}(\xi), \\
w_{2}(0)= & 0, \\
w_{2}(1)= & 0
\end{aligned}
$$

The solution of (19) is

$$
w_{2}(\xi)=0
$$

And the third-order approximate solution of the bvp (14) is as follows:

$$
\begin{aligned}
& w^{(2)}(\xi)=w_{0}(\xi)+w_{1}(\xi)+w_{2}(\xi) \\
& w^{(2)}(\xi)=\frac{\left(e-e^{\xi}\right) \xi}{\xi}
\end{aligned}
$$

Table 1 shows the comparison between the exact solution and the approximate solution obtained by OHAM. Figure 1 of the solution also shows well agreement with the exact solution.
3.2. Example 2. Consider the nonlinear two-point boundary value problem [1] of the type

$$
\begin{aligned}
w^{\prime \prime}(\xi) & =w^{3}(\xi)-w(\xi) w^{\prime}(\xi), \quad \xi \in[1,2], \\
w(1) & =\frac{1}{2} \\
w(2) & =\frac{1}{3}
\end{aligned}
$$

According to OHAM $\mathscr{L}(w(x))=w^{\prime \prime}(\xi)$ and $\mathbb{N}(w(\xi))=$ $\mathbf{u}(\xi) \mathbf{u}^{\prime}(\xi)-w^{3}(\xi)$, while $F(\xi)=0$. The exact solution of (23) is $1 /(\xi+1)$. Now proceeding with the same lines as above we have the following zeroth-order problem:

$$
\begin{aligned}
& w_{0}^{\prime \prime}(\xi)=0, \\
& w_{0}(1)=\frac{1}{2}, \\
& w_{0}(2)=\frac{1}{3}
\end{aligned}
$$

The solution of (24) is

$$
w_{0}(\xi)=\frac{4-\xi}{6}
$$

Now the first-order problem is

$$
\begin{aligned}
w_{1}^{\prime \prime}(\xi)= & \mathbb{C}_{1} w_{0}^{3}(\xi)-\mathbb{C}_{1} w_{0}(\xi) w_{0}^{\prime}(\xi)-w_{0}^{\prime \prime}(\xi) \\
& -\mathbb{C}_{1} w_{0}^{\prime \prime}(\xi) \\
w_{1}(1)= & 0 \\
w_{1}(2)= & 0
\end{aligned}
$$

The solution of (26) is

$$
\begin{aligned}
& w_{1}(\xi) \\
& =\frac{-930 \mathbb{C}_{1}+1649 \xi \mathbb{C}_{1}-880 \xi_{1}^{2} \mathbb{C}+180 \xi_{1}^{3} \mathbb{C}-20 \xi_{1}^{4} \mathbb{C}+\xi_{5 \mathbb{C} 1}}{4320}
\end{aligned}
$$

The second-order problem is

$$
\begin{aligned}
w_{2}^{\prime \prime}(\xi)= & \mathbb{C}_{2} w_{0}^{3}(\xi)+3 \mathbb{C}_{1} w_{0}^{2}(\xi) w_{1}(\xi) \\
& -\mathbb{C}_{2} w_{0}(\xi) w_{0}^{\prime}(\xi)-\mathbb{C}_{1} w_{1}(\xi) w_{0}^{\prime}(\xi) \\
& -\mathbb{C}_{1} w_{0}(\xi) w_{1}^{\prime}(\xi)-\mathbb{C}_{2} w_{0}^{\prime \prime}(\xi)-w_{1}^{\prime \prime}(\xi) \\
& -\mathbb{C}_{1} w_{1}^{\prime \prime}(\xi), \\
w_{2}(1)= & 0, \\
w_{2}(2)= & 0 .
\end{aligned}
$$


TABLE 2: Comparison of second-order OHAM solution with the exact solution for example 2.

\begin{tabular}{lccr}
\hline$\xi$ & OHAM Solution $\left(w^{(3)}\right)$ & Exact & $\mid w^{(3)}-E^{2}$ \\
\hline 1.1 & 0.47619 & 0.47619 & $2.0597 \times 10^{-7}$ \\
\hline 1.3 & 0.434783 & 0.434783 & $5.38284 \times 10^{-7}$ \\
\hline 1.5 & 0.400001 & 0.4 & $1.39261 \times 10^{-6}$ \\
\hline 1.7 & 0.370371 & 0.37037 & $6.7426 \times 10^{-7}$ \\
\hline 1.9 & 0.344828 & 0.344828 & $8.10196 \times 10^{-8}$ \\
\hline
\end{tabular}

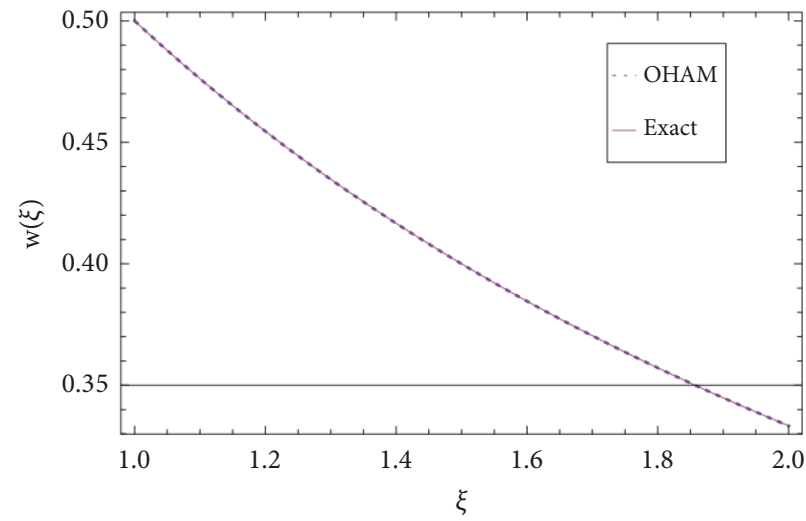

Figure 2: Comparison between exact solution (dashed line) and approximate solution (dotted line) for example 2.

The solution of (29) is

$$
\begin{aligned}
& w_{2}(\xi)=\frac{1}{130636800}(\xi-2)(\xi-1)\left\{3 0 2 4 0 \left(-465+\xi(127+(\xi-17) \xi) \mathbb{C}_{1}\right.\right. \\
& -(8985375+\xi(4253423+\xi(-3664113+\xi(1100519+5 \xi(-36795+\xi(3709+7(-33+\xi) \xi)))))) \mathbb{C}_{1}^{2} \\
& \left.\quad+30240(-465+\xi(127+(-17+\xi) \xi)) \mathbb{C}_{2}\right)
\end{aligned}
$$

The third-order problem is

$$
\begin{aligned}
\mathbb{C}_{3} w_{0}^{3}(\xi)+3 \mathbb{C}_{2} w_{0}^{2}(\xi) w_{1}(\xi)+3 \mathbb{C}_{1} w_{0}(\xi) w_{1}^{2}(\xi) \\
+3 \mathbb{C}_{1} w_{0}^{2}(\xi) w_{2}(\xi)-3 \mathbb{C}_{3} w_{0}(\xi) w_{0}^{\prime}(\xi) \\
-\mathbb{C}_{2} w_{1}(\xi) w_{0}^{\prime}(\xi)-\mathbb{C}_{1} w_{2}(\xi) w_{0}^{\prime}(\xi) \\
-\mathbb{C}_{2} w_{0}(\xi) w_{1}^{\prime}(\xi)-\mathbb{C}_{1} w_{1}(\xi) w_{1}^{\prime}(\xi) \\
-\mathbb{C}_{1} w_{0}(\xi) w_{2}^{\prime}(\xi)-\mathbb{C}_{3} w_{0}^{\prime \prime}(\xi)-\mathbb{C}_{2} w_{1}^{\prime \prime}(\xi) \\
-w_{2}^{\prime \prime}(\xi)-\mathbb{C}_{1} w_{2}^{\prime \prime}(\xi)+w_{3}^{\prime \prime}(\xi)=0
\end{aligned}
$$

The solution of the third-order problem results a large output, therefore not included here.

Now the third-order approximate solution is

$$
w^{(3)}(\xi)=w_{0}(\xi)+w_{1}(\xi)+w_{2}(\xi)+w_{3}(\xi)
$$

$\mathbb{C}_{i}^{\prime} s$ has the following values and then substituting in the above solution we will get the approximate solution. $w^{(3)}(\xi)$ is given in Appendix (A.1).

$$
\begin{aligned}
& \mathbb{C}_{1}=-0.9637924142971654 \\
& \mathbb{C}_{2}=-0.0002296939939480446 \\
& \mathbb{C}_{3}=-0.000014314891134337846
\end{aligned}
$$

The solution at the points given in Table 2 and the graph of the solution is shown in Figure 2. Here it is third-order OHAM solution while the HPM [1] gives the accuracy up to 9 decimal places in 7 th order.

3.3. Example 3. Now we consider higher order TPBVP of order four. The problem is

$$
\frac{d^{4} w(\xi)}{d \xi^{4}}=w^{2}(\xi)+\digamma(\xi), \quad 0 \leq \xi \leq 1
$$

with the boundary conditions $w(0)=0, w^{\prime}(0)=0, w(1)=1$, and $w^{\prime}(1)=1$. 
TABLE 3: Comparison of second-order OHAM solution with the exact solution for example 3.

\begin{tabular}{lccr}
\hline$\xi$ & OHAM Solution $\left(w^{(2)}\right)$ & Exact & $\mid w^{(2)}-$ Exact $\mid$ \\
\hline 0.2 & 0.077119 & 0.0771200 & $6.152306 \times 10^{-11}$ \\
0.4 & 0.279039 & 0.2790400 & $1.314346 \times 10^{-10}$ \\
0.6 & 0.538559 & 0.5385600 & $1.001054 \times 10^{-10}$ \\
0.8 & 0.788479 & 0.7884800 & $2.415356 \times 10^{-11}$ \\
\hline
\end{tabular}

Where $\mathscr{L}(w(\xi))=d^{4} w(\xi) / d x^{4}, \mathbb{N}(w(\xi))=w^{2}(\xi)$, and $F(\xi)=-\xi^{10}+4 \xi^{9}-4 \xi^{8}-4 \xi^{7}+8 \xi^{6}-4 \xi^{4}+120 \xi-48$, the exact solution of problem (34) is $w_{\text {exact }}=\xi^{5}-2 \xi^{4}+2 \xi^{2}$. After solving this by the method described in Section 2, we have the following zeroth-order problem:

$$
\begin{aligned}
& 48-120 \xi+4 \xi^{4}-8 \xi^{6}+4 \xi^{7}+4 \xi^{8}-4 \xi^{9}+\xi^{10} \\
& +\frac{d^{4} w_{0}(\xi)}{d \xi^{4}}=0 \\
& w_{0}(0)=0, \\
& w_{0}^{\prime}(0)=0, \\
& w_{0}(1)=1, \\
& w_{0}^{\prime}(1)=1
\end{aligned}
$$

The solution to (35) is

$$
\begin{aligned}
& w_{0}(\xi)=\frac{1}{1081080}\left(2155683 \xi^{2}+8038 \xi^{3}-2162160 \xi^{4}\right. \\
& \quad+1081080 \xi^{5}-2574 \xi^{8}+1716 \xi^{10}-546 \xi^{11} \\
& \left.\quad-364 \xi^{12}+252 \xi^{13}-45 \xi^{14}\right)
\end{aligned}
$$

The first-order problem is

$$
\begin{aligned}
& -48+120 \xi-4 \xi^{4}+8 \xi^{6}-4 \xi^{7}-4 \xi^{8}+4 \xi^{9}-\xi^{10} \\
& -48 \mathbb{C}_{1}+120 \xi \mathbb{C}_{1}-4 \xi_{1}^{4} \mathbb{C}+8 \xi^{6} \mathbb{C}_{1}-4 \xi^{7} \mathbb{C}_{1} \\
& -4 \xi^{8} \mathbb{C}_{1}+4 \xi^{9} \mathbb{C}_{1}-\xi^{10} \mathbb{C}_{1}+\mathbb{C}_{1} w_{0}^{2}(\xi) \\
& \quad-\left(1+\mathbb{C}_{1}\right) \frac{d^{4} w_{0}(\xi)}{d \xi^{4}}+\frac{d^{4} w_{1}(\xi)}{d \xi^{4}}=0 \\
& w_{1}(0)=0 \\
& w_{1}^{\prime}(0)=0 \\
& w_{1}(1)=0 \\
& w_{1}^{\prime}(1)=0 .
\end{aligned}
$$

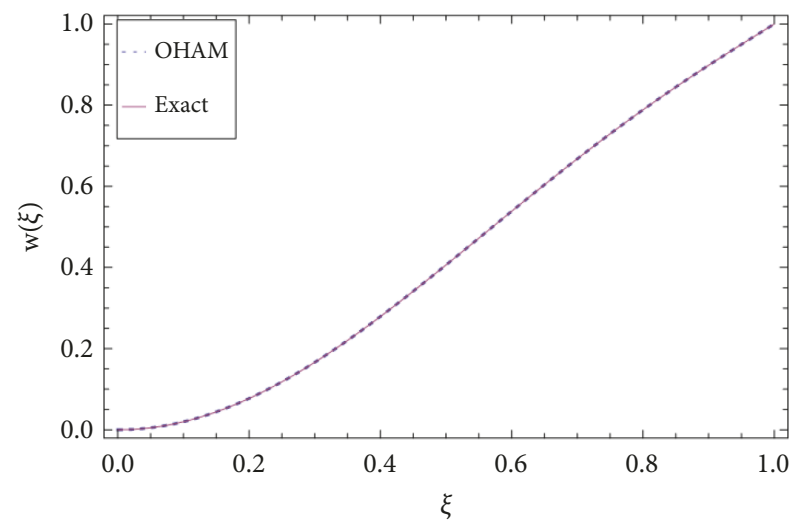

Figure 3: Comparison between exact solution (dashed line) and approximate solution (dotted line) for example 3.

The second-order problem is

$$
\begin{aligned}
& -48 \mathbb{C}_{2}+120 \xi \mathbb{C}_{2}-4 \xi^{4} \mathbb{C}_{2}+8 \xi^{6} \mathbb{C}_{2}-4 \xi^{7} \mathbb{C}_{2}-4 \xi^{8} \mathbb{C}_{2} \\
& +4 \xi^{9} \mathbb{C}_{2}-\xi^{10} \mathbb{C}_{2}+\mathbb{C}_{2} w_{0}^{2}(\xi)+2 \mathbb{C}_{1} w_{0}(\xi) w_{1}(\xi) \\
& -\mathbb{C}_{2} w_{0}^{(4)}(\xi)-w_{1}^{(4)}(\xi)-\mathbb{C}_{1} w_{1}^{(4)}(\xi)+w_{2}^{(4)}(\xi)=0 \\
& w_{2}(0)=0, \\
& w_{2}^{\prime}(0)=0, \\
& w_{2}(1)=0, \\
& w_{2}^{\prime}(1)=0 .
\end{aligned}
$$

The solutions of problem (38) and (40) are very large; therefore we did not write it here. The constants $\mathbb{C}_{1}$ and $\mathbb{C}_{2}$ have the values -1.0011320722175725 and $-1.079468959963785 \times$ $10^{-6}$, respectively. Table 3 and Figure 3 show a good agreement with the exact values. The approximate solution $w_{2}(\xi)$ is given in Appendix (A.2).

3.4. Example 4. At last, consider the second-order nonlinear TPBVP[1]

$$
\begin{aligned}
& w(0)=0, \\
& w(1)=0 .
\end{aligned}
$$$$
w^{\prime \prime}(\xi)=w^{2}(\xi)+2 \pi^{2} \cos (2 \pi \xi)-\sin ^{2}(2 \pi \xi),
$$$$
0 \leq \xi \leq 1
$$ 
TABLE 4: Comparison of third-order OHAM solution with the exact solution.

\begin{tabular}{lccr}
\hline$\xi$ & OHAM Solution $\left(w^{(3)}\right)$ & Exact & $\left|w^{(3)}-E x a c t\right|$ \\
\hline 0.1 & 0.0954915 & 0.0954915 & $5.59262 \times 10^{-9}$ \\
\hline 0.3 & 0.654508 & 0.654508 & $1.23779 \times 10^{-8}$ \\
\hline 0.5 & 0.999999 & 1. & $1.51565 \times 10^{-8}$ \\
\hline 0.7 & 0.654508 & 0.654508 & $1.23779 \times 10^{-8}$ \\
\hline 0.9 & 0.0954915 & 0.0954915 & $5.59262 \times 10^{-9}$ \\
\hline
\end{tabular}

The exact solution of (42) is $\sin ^{2}(\pi \xi)$. Solving (42) by the method depicted in Section 2, we have the following zeroth order problem:

$$
\begin{aligned}
-2 \pi^{2} \cos (2 \pi \xi)+\sin ^{4}(\pi \xi)+w_{0}^{\prime \prime}(\xi) & =0, \\
w_{0}(0) & =0 \\
w_{0}(1) & =0
\end{aligned}
$$

The solution of (44) is given by

$$
\begin{gathered}
w_{0}(\xi)=\frac{1}{128 \pi^{2}}\left(15-64 \pi^{2}+24 \pi^{2} \xi-24 \pi^{2} \xi^{2}\right. \\
\left.-16 \cos (2 \pi \xi)-64 \pi^{2} \cos (2 \pi \xi)+\cos (4 \pi \xi)\right)
\end{gathered}
$$

The first-, second-, and third-order problems are given in (46), (47), and (48) respectively.

$$
\begin{aligned}
& 2 \pi^{2} \cos (2 \pi \xi)\left(1+\mathbb{C}_{1}\right)-\sin ^{4}(\pi \xi)\left(1+\mathbb{C}_{1}\right) \\
& +\mathbb{C}_{1} w_{0}^{2}(\xi)-w_{0}^{\prime \prime}(\xi)-\mathbb{C}_{1} w_{0}^{\prime \prime}(\xi)+w_{1}^{\prime \prime}(\xi)=0, \\
& w_{1}(0)=0 \\
& w_{1}(1)=0 \\
& \left\{2 \pi^{2} \cos (2 \pi \xi)-\sin ^{4}(\pi \xi)\right\} \mathbb{C}_{2}+\mathbb{C}_{2} w_{0}^{2}(\xi) \\
& \quad+2 \mathbb{C}_{1} w_{0}(\xi) w_{1}(\xi)-\mathbb{C}_{2} w_{0}^{\prime \prime}(\xi)-w_{1}^{\prime \prime}(\xi) \\
& \quad-\mathbb{C}_{1} w_{1}^{\prime \prime}(\xi)+w_{2}^{\prime \prime}(\xi)=0, \\
& w_{2}(0)=0 \\
& w_{2}(1)=0 \\
& \left\{2 \pi^{2} \cos (2 \pi \xi)-\sin ^{4}(\pi \xi)\right\} \mathbb{C}_{3}+\mathbb{C}_{3} w_{0}^{2}(\xi) \\
& \quad+2 \mathbb{C}_{2} w_{0}(\xi) w_{1}(\xi)+\mathbb{C}_{1} w_{1}^{2}(\xi)+2 \mathbb{C}_{1} w_{0}(\xi) w_{2}(\xi) \\
& +\mathbb{C}_{3} w_{0}^{\prime \prime}(\xi)-\mathbb{C}_{2} w_{1}^{\prime \prime}(\xi)-w_{2}^{\prime \prime}(\xi)-\mathbb{C}_{1} w_{2}^{\prime \prime}(\xi) \\
& \quad+w_{3}^{\prime \prime}(\xi)=0
\end{aligned}
$$

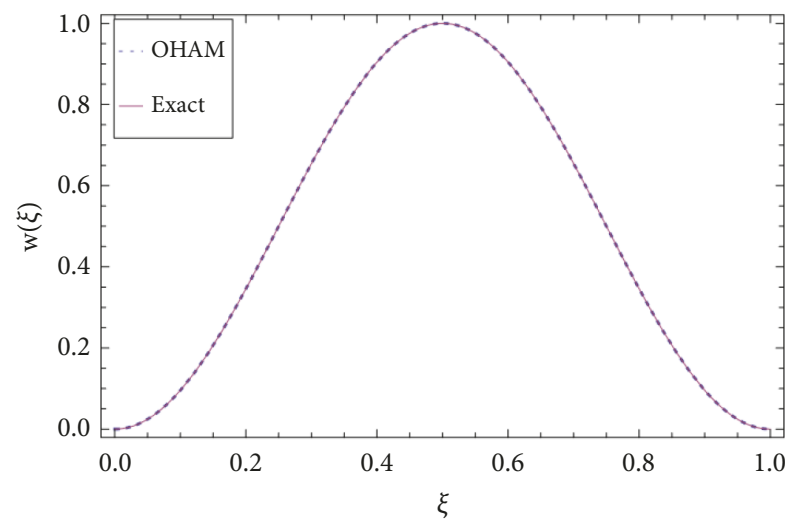

Figure 4: Comparison between exact solution (dashed line) and approximate solution (dotted line) for example 4.

$$
\begin{aligned}
& w_{3}(0)=0, \\
& w_{3}(1)=0
\end{aligned}
$$

The solutions of problem (46), (47), and (48) are very large and therefore cannot be written here but the table of values and the graph are shown in Table 4 and Figure 4, respectively. The approximate solution $w^{(3)}(\xi)$ is written in Appendix (A.3). The values of the constants $\mathbb{C}_{i}^{\prime} s$ can be found by (13) which are given as follows:

$$
\begin{aligned}
& \mathbb{C}_{1}=-0.9030981665320986 \\
& \mathbb{C}_{2}=-0.00569345107292796 \\
& \mathbb{C}_{3}=0.00021560218552318884
\end{aligned}
$$

\section{Conclusion}

This paper reveals that OHAM is a very strong method for solving TPBVP and gives us a more accurate solution as compared to other methods. In these examples only second- and third-order solution gives us the accuracy up to 8 or 10 decimal places; therefore it is concluded that this method converges very fast to the exact solution and in some problems like example 1 it gives us the exact solution. The plots and tables show well agreement with the exact solution. 


\section{Appendix}

$\stackrel{(3)}{w}(\xi)=\frac{4-\xi}{6}+\frac{1}{4320}\left(-930 \mathbb{C}_{1}+1649 \xi \mathbb{C}_{1}-880 \xi^{2} \mathbb{C}_{1}+180 \xi^{3} \mathbb{C}_{1}-20 \xi^{4} \mathbb{C}_{1}+\xi^{5} \mathbb{C}_{1}\right)+\frac{1}{130636800}\left(-28123200 \mathbb{C}_{1}\right.$

$+49865760 \xi \mathbb{C}_{1}-26611200 \xi^{2} \mathbb{C}_{1}+5443200 \xi^{3} \mathbb{C}_{1}-604800 \xi^{4} \mathbb{C}_{1}+30240 \xi^{5} \mathbb{C}_{1}-17970750 \mathbb{C}_{1}^{2}+18449279 \xi \mathbb{C}_{1}^{2}$

$+11103120 \xi^{2} \mathbb{C}_{1}^{2}-17446800 \xi^{3} \mathbb{C}_{1}^{2}+7333620 \xi^{4} \mathbb{C}_{1}^{2}-1689534 \xi^{5} \mathbb{C}_{1}^{2}+241920 \xi^{6} \mathbb{C}_{1}^{2}-22080 \xi^{7} \mathbb{C}_{1}^{2}+1260 \xi^{8} \mathbb{C}_{1}^{2}$

$\left.-35 \xi^{8} \mathbb{C}_{1}^{2}-28123200 \mathbb{C}_{2}+49865760 \xi \mathbb{C}_{2}-26611200 \xi^{2} \mathbb{C}_{2}+5443200 \xi^{3} \mathbb{C}_{2}-604800 \xi^{4} \mathbb{C}_{2}+30240 \xi^{5} \mathbb{C}_{2}\right)$

$+\frac{1}{13450364928000}\left(-2895564672000 \mathbb{C}_{1}+5134178649600 \xi \mathbb{C}_{1}-2739889152000 \xi^{2} \mathbb{C}_{1}+560431872000 \xi^{3} \mathbb{C}_{1}\right.$

$-62270208000 \xi^{4} \mathbb{C}_{1}+3113510400 \xi^{5} \mathbb{C}_{1}-3700536840000 \mathbb{C}_{1}^{2}+3799075531680 \xi \mathbb{C}_{1}^{2}+2286354470400 \xi^{2} \mathbb{C}_{1}^{2}$

$-3592645056000 \xi^{3} \mathbb{C}_{1}^{2}+1510139030400 \xi^{4} \mathbb{C}_{1}^{2}-347908841280 \xi^{5} \mathbb{C}_{1}^{2}+49816166400 \xi^{6} \mathbb{C}_{1}^{2}-4546713600 \xi^{7} \mathbb{C}_{1}^{2}$

$+259459200 \xi^{8} \mathbb{C}_{1}^{2}-7207200 \xi^{9} \mathbb{C}_{1}^{2}-1161287826270 \mathbb{C}_{1}^{3}+270053629823 \xi \mathbb{C}_{1}^{3}+1988069792280 \xi^{2} \mathbb{C}_{1}^{3}$

$-963479454080 \xi^{3} \mathbb{C}_{1}^{3}-507564676190 \xi^{4} \mathbb{C}_{1}^{3}+549609764847 \xi^{5} \mathbb{C}_{1}^{3}-221549328000 \xi^{6} \mathbb{C}_{1}^{3}+54219285120 \xi^{7} \mathbb{C}_{1}^{3}$

$-9069733530 \xi^{8} \mathbb{C}_{1}^{3}+1086825025 \xi^{9} \mathbb{C}_{1}^{3}-93716480 \xi^{10} \mathbb{C}_{1}^{3}+5653440 \xi^{11} \mathbb{C}_{1}^{3}-220220 \xi^{12} \mathbb{C}_{1}^{3}+4235 \xi^{13} \mathbb{C}_{1}^{3}$

$-2895564672000 \mathbb{C}_{2}+5134178649600 \xi \mathbb{C}_{2}-2739889152000 \xi^{2} \mathbb{C}_{2}+560431872000 \xi^{3} \mathbb{C}_{2}-62270208000 \xi^{4} \mathbb{C}_{2}$

$+3113510400 \xi^{5} \mathbb{C}_{2}-3700536840000 \mathbb{C}_{1} \mathbb{C}_{2}+3799075531680 \xi \mathbb{C}_{1} \mathbb{C}_{2}+2286354470400 \xi^{2} \mathbb{C}_{1} \mathbb{C}_{2}$

$-3592645056000 \xi^{3} \mathbb{C}_{1} \mathbb{C}_{2}+1510139030400 \xi^{4} \mathbb{C}_{1} \mathbb{C}_{2}-347908841280 \xi^{5} \mathbb{C}_{1} \mathbb{C}_{2}+49816166400 \xi^{6} \mathbb{C}_{1} \mathbb{C}_{2}$

$-4546713600 \xi^{7} \mathbb{C}_{1} \mathbb{C}_{2}+259459200 \xi^{8} \mathbb{C}_{1} \mathbb{C}_{2}-7207200 \xi^{9} \mathbb{C}_{1} \mathbb{C}_{2}-2895564672000 \mathbb{C}_{3}+5134178649600 \xi \mathbb{C}_{3}$

$\left.-2739889152000 \xi^{2} \mathbb{C}_{3}+560431872000 \xi^{3} \mathbb{C}_{3}-62270208000 \xi^{4} \mathbb{C}_{3}+3113510400 \xi^{5} \mathbb{C}_{3}\right)$.

$w^{(2)}(\xi)=\left(2155683 \xi^{2}+8038 \xi^{3}-2162160 \xi^{4}+1081080 \xi^{5}-2574 \xi^{8}+1716 \xi^{10}-546 \xi^{11}-364 \xi^{12}+252 \xi^{13}-45 \xi^{14}\right) /$

$1081080+1 / 2360410309588661890560000\left(-14113828503813453911359 \xi^{2} \mathbb{C}_{1}\right.$

$+17512915766704666962322 \xi^{3} \mathbb{C}_{1}-5586404113887189501420 \xi^{8} \mathbb{C}_{1}-23144774000952226800 \xi^{9} \mathbb{C}_{1}$

$+3735433519034813810160 \xi^{10} \mathbb{C}_{1}-1179691619002973294400 \xi^{11} \mathbb{C}_{1}-797705495192034374400 \xi^{12} \mathbb{C}_{1}$

$+550212193377310464000 \xi^{13} \mathbb{C}_{1}-97319245468388853600 \xi^{14} \mathbb{C}_{1}+2551023644304960 \xi^{15} \mathbb{C}_{1}$

$-856729299998872080 \xi^{16} \mathbb{C}_{1}+279036341094724200 \xi^{17} \mathbb{C}_{1}+247466505045614400 \xi^{18} \mathbb{C}_{1}$

$-155275084044250800 \xi^{19} \mathbb{C}_{1}-3650519253533040 \xi^{2} 0 \mathbb{C}_{1}+26398020790188000 \xi^{2} 1 \mathbb{C}_{1}$

$-8405056667479680 \xi^{2} 2 \mathbb{C}_{1}+897927547083840 \xi^{2} 3 \mathbb{C}_{1}-38159719228800 \xi^{2} 4 \mathbb{C}_{1}+21095475553152 \xi^{2} 5 \mathbb{C}_{1}$

$+4049797421376 \xi^{2} 6 \mathbb{C}_{1}-6052906241984 \xi^{2} 7 \mathbb{C}_{1}+1221181635008 \xi^{2} 8 \mathbb{C}_{1}+475889853600 \xi^{2} 9 \mathbb{C}_{1}$

$\left.-295593372480 \xi^{3} 0 \mathbb{C}_{1}+60656299200 \xi^{3} 1 \mathbb{C}_{1}-4738773375 \xi^{3} 2 \mathbb{C}_{1}\right)$

$+\left(\left(-32669682535166038177800562717403451774787200 \xi^{2} \mathbb{C}_{1}\right.\right.$

+ $40537647046565498276872580694218893070457600 \xi^{3} \mathbb{C}_{1}$

- $12931009390154359168924266696920562467136000 \xi^{8} \mathbb{C}_{1}$ 
$+40537647046565498276872580694218893070457600 \xi^{3} \mathbb{C}_{1}$

- $12931009390154359168924266696920562467136000 \xi^{8} \mathbb{C}_{1}$

- $53573870389240769630688977156787421440000 \xi^{9} \mathbb{C}_{1}$

$+8646514810996349122418420307721339216128000 \xi^{10} \mathbb{C}_{1}$

- $2730665933188143522672522412342587755520000 \xi^{11} \mathbb{C}_{1}$

- $1846471726465979561236202970676720619520000 \xi^{12} \mathbb{C}_{1}$

$+1273591901712376237386288026597835571200000 \xi^{13} \mathbb{C}_{1}$

- $225267641104971069200162200032882362880000 \xi^{14} \mathbb{C}_{1}+5904927396321076366168727485651968000 \xi^{15} \mathbb{C}_{1}$

- $1983095815708383589080913455027281664000 \xi^{16} \mathbb{C}_{1}+645893399999572047403581539117439360000 \xi^{17} \mathbb{C}_{1}$

$+572817797505678403219571120181611520000 \xi^{18} \mathbb{C}_{1}$

$+8620466886846280020965176767572231570851512 \xi^{10} \mathbb{C}_{1}^{2}$

$-2701942454241603867437283593123940978268560 \xi^{11} \mathbb{C}_{1}^{2}$

- $1853296246170788567680120913554535247983040 \xi^{12} \mathbb{C}_{1}^{2}$

$+1273591901712376237386288026597835571200000 \xi^{13} \mathbb{C}_{1}^{2}$

$-223127551098503747806099303888254244271880 \xi^{14} \mathbb{C}_{1}^{2}+24188753111272816253620159558815649776 \xi^{15} \mathbb{C}_{1}^{2}$

- $3954294999119289026707499780027151742404 \xi^{16} \mathbb{C}_{1}^{2}+1280476731768992936554600505857043216034 \xi^{17} \mathbb{C}_{1}^{2}$

$+1146299848262837493979094855639474837488 \xi^{18} \mathbb{C}_{1}^{2}-716583570253989121237950089315154553020 \xi^{19} \mathbb{C}_{1}^{2}$

$-18153410829102066767079156365109506436 \xi^{2} 0 \mathbb{C}_{1}^{2}+122325062475997255309755518897728337880 \xi^{2} 1 \mathbb{C}_{1}^{2}$

$-38583478912638559610674295035606805760 \xi^{2} 2 \mathbb{C}_{1}^{2}+4064708510722834491402346035781177920 \xi^{2} 3 \mathbb{C}_{1}^{2}$

$-304944482768990447155003185921595200 \xi^{2} 4 \mathbb{C}_{1}^{2}+172131824316102518718368542082822400 \xi^{2} 5 \mathbb{C}_{1}^{2}$

$+31225989769228784108742227282106504 \xi^{2} 6 \mathbb{C}_{1}^{2}-48696064947262435404352469277878496 \xi^{2} 7 \mathbb{C}_{1}^{2}$

+ $9955954110432674815496294613892416 \xi^{2} 8 \mathbb{C}_{1}^{2}+3768051722420250956380935649060800 \xi^{2} 9 \mathbb{C}_{1}^{2}$

$-2341220030385245677826756247386880 \xi^{3} 0 \mathbb{C}_{1}^{2}+474526294591146486759733985232768 \xi^{3} 1 \mathbb{C}_{1}^{2}$

$-40917013297075779490615187391192 \xi^{3} 2 \mathbb{C}_{1}^{2}+3867571077895433794192598216016 \xi^{3} 3 \mathbb{C}_{1}^{2}$

$-521615945593005771502089327960 \xi^{3} 4 \mathbb{C}_{1}^{2}-578651890632287750071052046372 \xi^{3} 5 \mathbb{C}_{1}^{2}$

$+265019543551924982873921756160 \xi^{3} 6 \mathbb{C}_{1}^{2}-7980251045802826679782141248 \xi^{3} 7 \mathbb{C}_{1}^{2}$

$-25268923385823287170976713452 \xi^{3} 8 \mathbb{C}_{1}^{2}+8700984701390689923286015560 \xi^{3} 9 \mathbb{C}_{1}^{2}$

$-1340309891030782820607242784 \xi^{4} 0 \mathbb{C}_{1}^{2}+135045166608803637462708432 \xi^{4} 1 \mathbb{C}_{1}^{2}$

$-18683464135506877900207872 \xi^{4} 2 \mathbb{C}_{1}^{2}-3279443942818588507522560 \xi^{4} 3 \mathbb{C}_{1}^{2}$

$+3820823675617325912753400 \xi^{4} 4 \mathbb{C}_{1}^{2}-805194420243411190696704 \xi^{4} 5 \mathbb{C}_{1}^{2}-143582548822550108696880 \xi^{4} 6 \mathbb{C}_{1}^{2}$

$+115436320975091250290520 \xi^{4} 7 \mathbb{C}_{1}^{2}-27795688158510545773500 \xi^{4} 8 \mathbb{C}_{1}^{2}+3304269870772182097500 \xi^{4} 9 \mathbb{C}_{1}^{2}$ 
- $165213493538609104875 \xi^{5} 0 \mathbb{C}_{1}^{2}-32669682535166038177800562717403451774787200 \xi^{2} \mathbb{C}_{2}$

$+40537647046565498276872580694218893070457600 \xi^{3} \mathbb{C}_{2}$

- $12931009390154359168924266696920562467136000 \xi^{8} \mathbb{C}_{2}$

- $53573870389240769630688977156787421440000 \xi^{9} \mathbb{C}_{2}$

$+8646514810996349122418420307721339216128000 \xi^{10} \mathbb{C}_{2}$

- $2730665933188143522672522412342587755520000 \xi^{11} \mathbb{C}_{2}$

- $1846471726465979561236202970676720619520000 \xi^{12} \mathbb{C}_{2}$

$+1273591901712376237386288026597835571200000 \xi^{13} \mathbb{C}_{2}$

- $225267641104971069200162200032882362880000 \xi^{14} \mathbb{C}_{2}+5904927396321076366168727485651968000 \xi^{15} \mathbb{C}_{2}$

- $1983095815708383589080913455027281664000 \xi^{16} \mathbb{C}_{2}+645893399999572047403581539117439360000 \xi^{17} \mathbb{C}_{2}$

$+572817797505678403219571120181611520000 \xi^{18} \mathbb{C}_{2}-359419678365531276056662133636240640000 \xi^{19} \mathbb{C}_{2}$

$-8449961331839350421935750500018432000 \xi^{2} 0 \mathbb{C}_{2}+611042537847995540864465470704550400000 \xi^{2} 1 \mathbb{C}_{2}$

$-19455425077784486158148965087647744000 \xi^{2} 2 \mathbb{C}_{2}+2078458576628113491233720925699072000 \xi^{2} 3 \mathbb{C}_{2}$

$-88329393580142122870344465623040000 \xi^{2} 4 \mathbb{C}_{2}+48830300656099081258580171252121600 \xi^{2} 5 \mathbb{C}_{2}$

+ $9374181927486220561295860381900800 \xi^{2} 6 \mathbb{C}_{2}-14010835209405620008882949076787200 \xi^{2} 7 \mathbb{C}_{2}$

$+2826704059972590662056252066406400 \xi^{2} 8 \mathbb{C}_{2}+1101555855990308389571183162880000 \xi^{2} 9 \mathbb{C}_{2}$

$-684218434127313503766165801984000 \xi^{3} 0 \mathbb{C}_{2}+140402870708442139347389199360000 \xi^{3} 1 \mathbb{C}_{2}$

- $\left.\left.10968974274097042136514781200000 \xi^{3} 2 \mathbb{C}_{2}\right)\right) / 5463709258346094058387175634104714600448000000$

$+\left(\left(-63812409443609717028183505295259001508810064369553098399996659827200000 \xi^{2} \mathbb{C}_{1}\right)\right) /$

1067204898300215968287533458623769753803390805870787878857474048000000000

$w^{(3)}(\xi)=\left(15+64 \pi^{2}+24 \pi^{2} \xi-24 \pi^{2} \xi^{2}-16 \cos [2 \pi \xi]-64 \pi^{2} \cos [2 \pi \xi]+\cos [4 \pi \xi]\right) /\left(128 \pi^{2}\right)+1 /\left(94371840 \pi^{6}\right)$

$\cdot\left(2312275 \mathbb{C}_{1}+12037120 \pi^{2} \mathbb{C}_{1}+11059200 \pi^{4} \mathbb{C}_{1}+1018080 \pi^{2} \xi \mathbb{C}_{1}+8824320 \pi^{4} \xi \mathbb{C}_{1}+19224576 \pi^{6} \xi \mathbb{C}_{1}\right.$

$-1018080 \pi^{2} \xi^{2} \mathbb{C}_{1}-8478720 \pi^{4} \xi^{2} \mathbb{C}_{1}-17694720 \pi^{6} \xi^{2} \mathbb{C}_{1}-691200 \pi^{4} \xi^{3} \mathbb{C}_{1}-2949120 \pi^{6} \xi^{3} \mathbb{C}_{1}+345600 \pi^{4} \xi^{4} \mathbb{C}_{1}$

$+1198080 \pi^{6} \xi^{4} \mathbb{C}_{1}+331776 \pi^{6} \xi^{5} \mathbb{C}_{1}-110592 \pi^{6} \xi^{6} \mathbb{C}_{1}-2373120 \cos [2 \pi \xi] \mathbb{C}_{1}-12441600 \pi^{2} \cos [2 \pi \xi] \mathbb{C}_{1}$

$-11796480 \pi^{4} \cos [2 \pi \xi] \mathbb{C}_{1}-1105920 \pi^{2} \xi \cos [2 \pi \xi] \mathbb{C}_{1}-4423680 \pi^{4} \xi \cos [2 \pi \xi] \mathbb{C}_{1}+1105920 \pi^{2} \xi^{2} \cos [2 \pi \xi] \mathbb{C}_{1}$

$+4423680 \pi^{4} \xi^{2} \cos [2 \pi \xi] \mathbb{C}_{1}+63360 \cos [4 \pi \xi] \mathbb{C}_{1}+414720 \pi^{2} \cos [4 \pi \xi] \mathbb{C}_{1}+737280 \pi^{4} \cos [4 \pi \xi] \mathbb{C}_{1}$

$+17280 \pi^{2} \xi \cos [4 \pi \xi] \mathbb{C}_{1}-17280 \pi^{2} \xi^{2} \cos [4 \pi \xi] \mathbb{C}_{1}-2560 \cos [6 \pi \xi] \mathbb{C}_{1}-10240 \pi^{2} \cos [6 \pi \xi] \mathbb{C}_{1}+45 \cos [8 \pi \xi] \mathbb{C}_{1}$

$+1105920 \pi \sin [2 \pi \xi] \mathbb{C}_{1}+4423680 \pi^{3} \sin [2 \pi \xi] \mathbb{C}_{1}-2211840 \pi \xi \sin [2 \pi \xi] \mathbb{C}_{1}-8847360 \pi^{3} \xi \sin [2 \pi \xi] \mathbb{C}_{1}$

$\left.-8640 \pi \sin [4 \pi \xi] \mathbb{C}_{1}+17280 \pi \xi \sin [4 \pi \xi] \mathbb{C}_{1}\right)+1 /\left(487049291366400 \pi^{10}\right)\left(11933558784000 \pi^{4} \mathbb{C}_{1}\right)+1 /$

$\left(35659800916682342400 \pi^{12}\right) \xi\left(384696643800268800 \pi^{8} \mathbb{C}_{1}+3334400329855795200 \pi^{10} \mathbb{C}_{1}\right.$

$+7264291475800719360 \pi^{12} \mathbb{C}_{1}+317056740153753600 \pi^{4} \mathbb{C}_{1}^{2}+3055348438990848000 \pi^{6} \mathbb{C}_{1}^{2}$ 


$$
\begin{aligned}
& +9761324410664386560 \pi^{8} \mathbb{C}_{1}^{2}+14367361192521891840 \pi^{10} \mathbb{C}_{1}^{2}+15851242925787709440 \pi^{12} \mathbb{C}_{1}^{2} \\
& +222124734074698455 \mathbb{C}_{1}^{3}+2171404189900081136 \pi^{2} \mathbb{C}_{1}^{3}+7100075693557310464 \pi^{4} \mathbb{C}_{1}^{3} \\
& +10842199196917637120 \pi^{6} \mathbb{C}_{1}^{3}+14250836120871567360 \pi^{8} \mathbb{C}_{1}^{3}+11649501141852487680 \pi^{10} \mathbb{C}_{1}^{3} \\
& +8684066594856370176 \pi^{12} \mathbb{C}_{1}^{3}+384696643800268800 \pi^{8} \mathbb{C}_{2}+3334400329855795200 \pi^{10} \mathbb{C}_{2} \\
& +7264291475800719360 \pi^{12} \mathbb{C}_{2}+317056740153753600 \pi^{4} \mathbb{C}_{1} \mathbb{C}_{2}+3055348438990848000 \pi^{6} \mathbb{C}_{1} \mathbb{C}_{2} \\
& +9761324410664386560 \pi^{8} \mathbb{C}_{1} \mathbb{C}_{2}+14367361192521891840 \pi^{10} \mathbb{C}_{1} \mathbb{C}_{2}+15851242925787709440 \pi^{12} \mathbb{C}_{1} \mathbb{C}_{2} \\
& \left.+384696643800268800 \pi^{8} \mathbb{C}_{3}+3334400329855795200 \pi^{10} \mathbb{C}_{3}+7264291475800719360 \pi^{12} \mathbb{C}_{3}\right)+1 / \\
& \left(3770462866155503616000000 \pi^{14}\right)\left(92382929312808960000000 \pi^{8} \mathbb{C}_{1}+480922211281010688000000 \pi^{10} \mathbb{C}_{1}\right. \\
& +441851117127598080000000 \pi^{12} \mathbb{C}_{1}+241156678171145748480000 \pi^{4} \mathbb{C}_{1}^{2}+1273402703047211089920000 \pi^{6} \mathbb{C}_{1}^{2} \\
& +1659580957335748608000000 \pi^{8} \mathbb{C}_{1}^{2}+1923688845124042752000000 \pi^{10} \mathbb{C}_{1}^{2} \\
& +883702234255196160000000 \pi^{12} \mathbb{C}_{1}^{2}+594085555282615418420429 \mathbb{C}_{1}^{3}+2862436440052784429431616 \pi^{2} \mathbb{C}_{1}^{3} \\
& +2597952006766286551449600 \pi^{4} \mathbb{C}_{1}^{3}+3066666484153535692800000 \pi^{6} \mathbb{C}_{1}^{3}+2139343103453036544000000 \pi^{8} \mathbb{C}_{1}^{3} \\
& +1442766633843032064000000 \pi^{10} \mathbb{C}_{1}^{3}+441851117127598080000000 \pi^{12} \mathbb{C}_{1}^{3}+92382929312808960000000 \pi^{8} \mathbb{C}_{2} \\
& +480922211281010688000000 \pi^{10} \mathbb{C}_{2}+441851117127598080000000 \pi^{12} \mathbb{C}_{2} \\
& +241156678171145748480000 \pi^{4} \mathbb{C}_{1} \mathbb{C}_{2}+1273402703047211089920000 \pi^{6} \mathbb{C}_{1} \mathbb{C}_{2} \\
& +1659580957335748608000000 \pi^{8} \mathbb{C}_{1} \mathbb{C}_{2}+1923688845124042752000000 \pi^{10} \mathbb{C}_{1} \mathbb{C}_{2} \\
& +883702234255196160000000 \pi^{12} \mathbb{C}_{1} \mathbb{C}_{2}+92382929312808960000000 \pi^{8} \mathbb{C}_{3}+480922211281010688000000 \pi^{10} \mathbb{C}_{3} \\
& \left.+441851117127598080000000 \pi^{12} \mathbb{C}_{3}\right)+1 /\left(124684618589798400 \pi^{12}\right)\left(-1345093160140800 \pi^{8} \xi^{2} \mathbb{C}_{1}\right)
\end{aligned}
$$

\section{Data Availability}

No data were used to support this study.

\section{Conflicts of Interest}

The authors declare that they have no conflicts of interest.

\section{References}

[1] C. Chun and R. Sakthivel, "Homotopy perturbation technique for solving two-point boundary value problems-comparison with other methods," Computer Physics Communications, vol. 181, no. 6, pp. 1021-1024, 2010.

[2] H. B. Keller, Numerical Methods for Two-Point Boundary-Value Problems, Courier Dover Publications, 2018.

[3] G. Adomian, "A review of the decomposition method in applied mathematics," Journal of Mathematical Analysis and Applications, vol. 135, no. 2, pp. 501-544, 1988.

[4] M. Tatari and M. Dehghan, "The use of the Adomian decomposition method for solving multipoint boundary value problems," Physica Scripta, vol. 73, no. 6, pp. 672-676, 2006.
[5] M. Inc and D. J. Evans, "The decomposition method for solving of a class of singular two-point boundary value problems," International Journal of Computer Mathematics, vol. 80, no. 7, pp. 869-882, 2003.

[6] B. Jang, "Exact solutions to one dimensional non-homogeneous parabolic problems by the homogeneous Adomian decomposition method," Applied Mathematics and Computation, vol. 186, no. 2, pp. 969-979, 2007.

[7] A. M. Wazwaz, "A reliable algorithm for obtaining positive solutions for nonlinear boundary value problems," Computers \& Mathematics with Applications, vol. 41, pp. 1237-1244, 2001.

[8] B. Jang, "Two-point boundary value problems by the extended Adomian decomposition method," Journal of Computational and Applied Mathematics, vol. 219, no. 1, pp. 253-262, 2008.

[9] H. Yaghoobi and M. Torabi, "The application of differential transformation method to nonlinear equations arising in heat transfer," International Communications in Heat and Mass Transfer, vol. 38, no. 6, pp. 815-820, 2011.

[10] D. D. Ganji, G. A. Afrouzi, and R. A. Talarposhti, "Application of variational iteration method and homotopy-perturbation method for nonlinear heat diffusion and heat transfer equations," Physics Letters Section A: General, Atomic and Solid State Physics, vol. 368, no. 6, pp. 450-457, 2007. 
[11] A. H. Nayfeh, Introduction to Perturbation Techniques, John Wiley \& Sons, New York, NY, USA, 1981.

[12] A. H. Nayfeh, Perturbation Methods, John Wiley \& Sons, New York, NY, USA, 2000.

[13] A. Rafiq, M. Y. Malik, and T. Abbasi, "Solution of nonlinear pull-in behavior in electrostatic micro-actuators by using He's homotopy perturbation method," Computers \& Mathematics with Applications. An International Journal, vol. 59, no. 8, pp. 2723-2733, 2010.

[14] V. Marinca and N. Herişanu, "Application of optimal homotopy asymptotic method for solving nonlinear equations arising in heat transfer," International Communications in Heat and Mass Transfer, vol. 35, no. 6, pp. 710-715, 2008.

[15] M. Javidi and A. Golbabai, "A new domain decomposition algorithm for generalized Burger's-Huxley equation based on Chebyshev polynomials and preconditioning," Chaos, Solitons \& Fractals, vol. 39, no. 2, pp. 849-857, 2009.

[16] M. Wang, D. Li, C. Zhang, and Y. Tang, "Long time behavior of solutions of gKdV equations," Journal of Mathematical Analysis and Applications, vol. 390, no. 1, pp. 136-150, 2012.

[17] A. Acosta, P. García, H. Leiva, and A. Merlitti, "Finite Time Synchronization of Extended Nonlinear Dynamical Systems Using Local Coupling," International Journal of Differential Equations, vol. 2017, Article ID 1946304, 7 pages, 2017.

[18] D. Li and J. Zhang, "Efficient implementation to numerically solve the nonlinear time fractional parabolic problems on unbounded spatial domain," Journal of Computational Physics, vol. 322, pp. 415-428, 2016.

[19] D. Li, J. Zhang, and Z. Zhang, "Unconditionally optimal error estimates of a linearized galerkin method for nonlinear time fractional reaction-subdiffusion equations," Journal of Scientific Computing, pp. 1-19, 2018.

[20] A. J. Khattak, "A computational meshless method for the generalized Burger's-Huxley equation," Applied Mathematical Modelling, vol. 33, no. 9, pp. 3718-3729, 2009. 


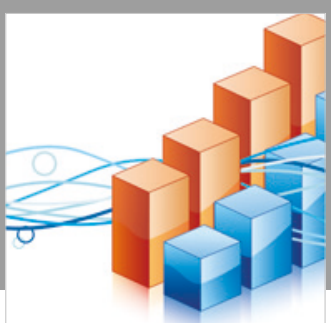

Advances in

Operations Research

\section{-n-m}
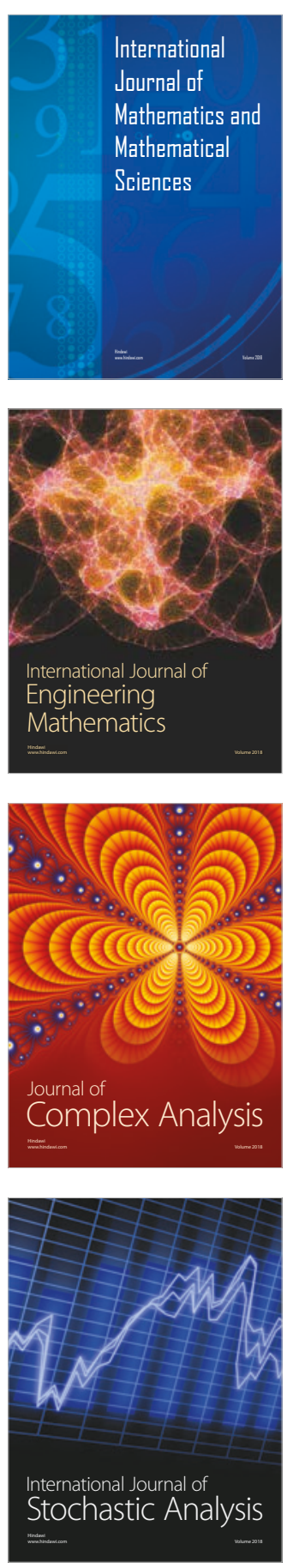
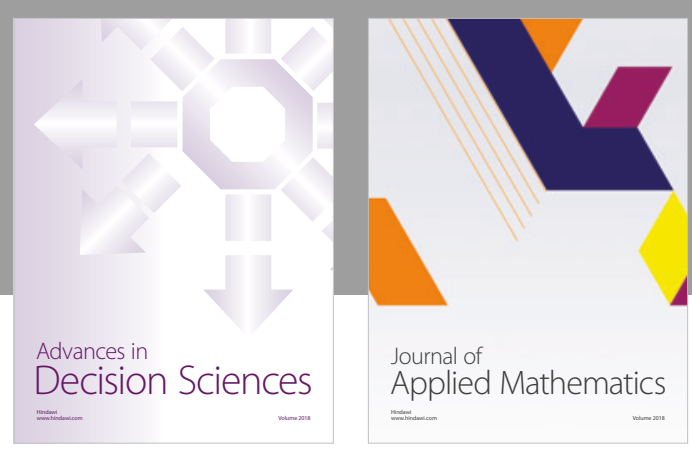

Journal of

Applied Mathematics
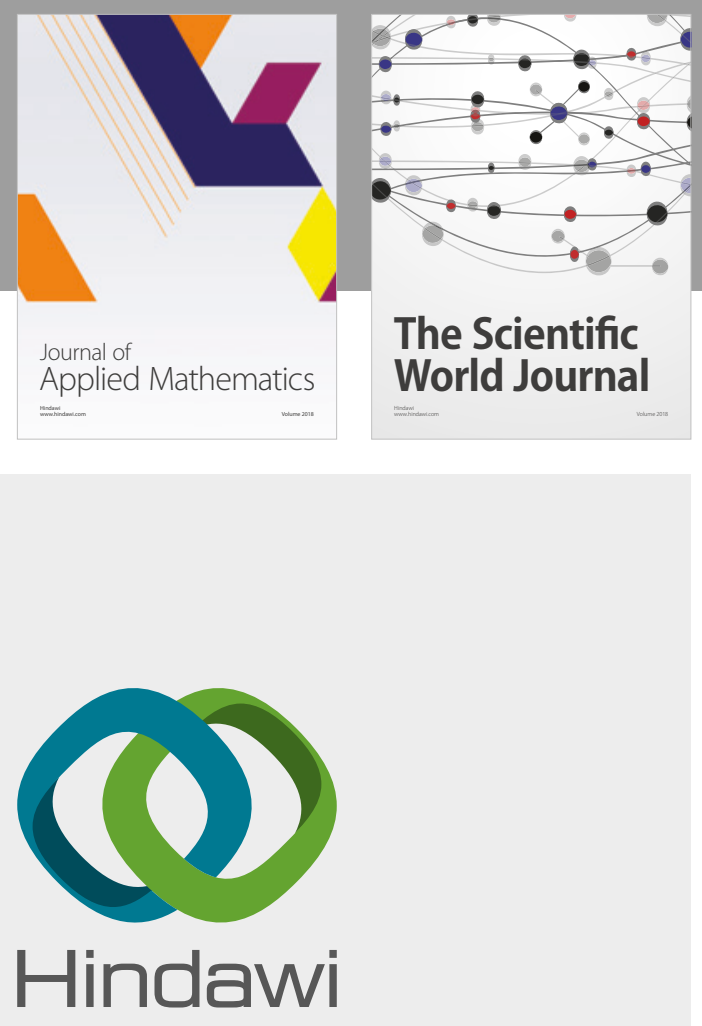

Submit your manuscripts at

www.hindawi.com

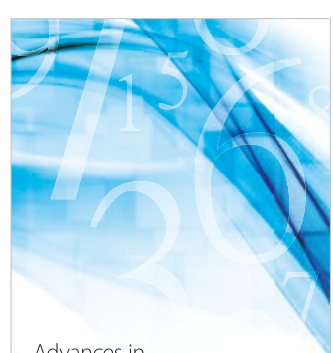

Advances in
Numerical Analysis
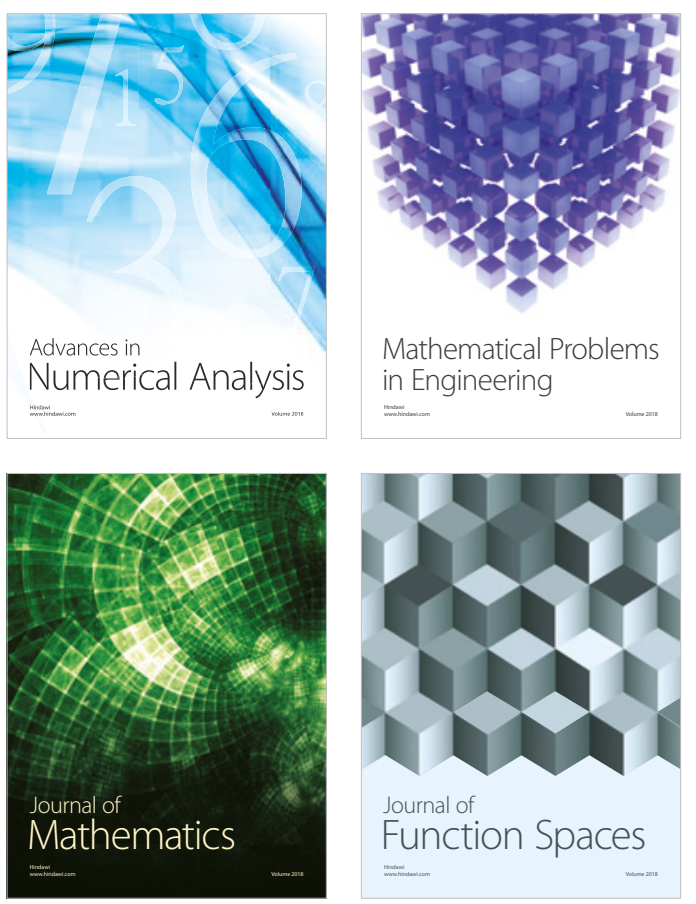

Mathematical Problems in Engineering

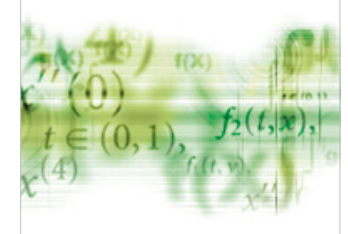

International Journal of

Differential Equations

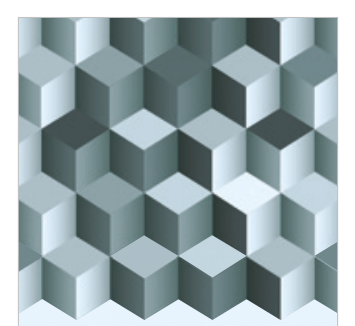

Journal of

Function Spaces

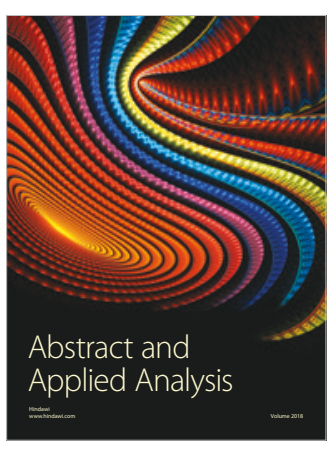

The Scientific

World Journal

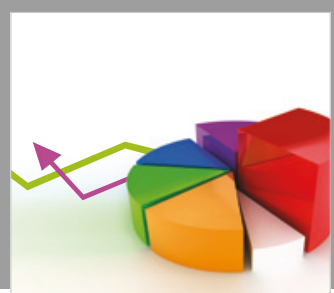

Journal of

Probability and Statistics
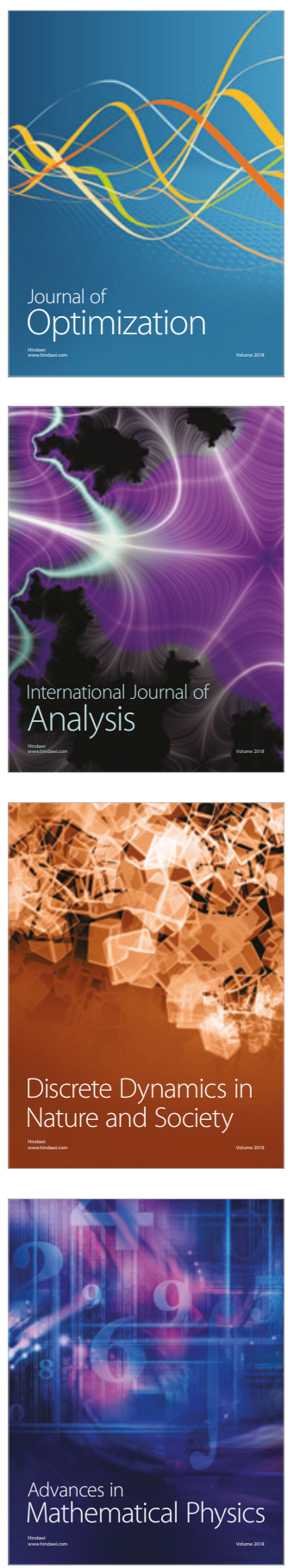